\section{New Evidence Showing yet another Link between Sight- Threatening Diabetic Retinopathy and Obstructive Sleep Apnea: How Relevant is this to my General Practice?}

Sir,

Obstructive sleep apnea (OSA) is a breathing disorder wherein the upper airways are intermittently blocked during sleep. ${ }^{1}$ It is a common complication in type 2 diabetes (T2D) but its potential effects on the other micro-vascular complications of diabetes, especially diabetic retinopathy (DR), are still unknown. DR is an ocular complication that can progress to sightthreatening diabetic retinopathy (STDR) that often causes blindness among the diabetics.2 At present, there is no consensus among the clinicians to screen diabetic patients for OSA due to lack of evidence on any correlation between OSA and DR progression. ${ }^{3}$

Interestingly, Altaf et al. hypothesised a mechanistic role of OSA in STDR pathogenesis due to the positive association of OSA with several pathophysiological features frequently seen in DR. They assessed $230 \mathrm{~T} 2 \mathrm{D}$ patients for DR using two-field 45-degree retinal images and for OSA using a multichannel cardiorespiratory device designed for home use. ${ }^{4}$ They reported STDR and OSA in $36.1 \%$ and $63.9 \%$ of the patients, respectively, and the prevalence of STDR in their study was higher than that reported earlier (5-15\%). ${ }^{4}$ Furthermore, the inclusion criteria disregarded the gender, race, as well as the severity of DR, resulting in a broader cohort of T2D patients that could be subjected to a longitudinal analysis, and present a stronger relationship between OSA and STDR.

Important mechanism is that during sleep, the retina is exposed to lower oxygen levels and susceptible to hypoxic damage. The latter is further exacerbated with accompanying OSA due to the high risk of hypoxemia, as supported by the observation that OSA patients had a significantly higher risk of progressing to DR (18.4\%) compared to those without OSA over a period of 43 months. ${ }^{4}$ Therefore, OSA is a likely independent predictor in T2D patients for the development of moderate to severe DR or STDR. Recent reports indicate that Pakistan has 35.3 million T2D cases, one of the highest in the world. ${ }^{5}$ Therefore, it is imperative that general practitioners (GPs) who serve as frontliners are aware of the risk of accompanying OSA in T2D. The GPs should be cognizant of a T2D patient's general health and habits such as snoring, day-time fatigue and sleepiness to determine any possible risk of OSA. For patients with uncontrolled T2D, early referral to a specialist can prevent subsequent STDR. It is also important to improve the communication between GPs and specialists vis-à-vis complications such as OSA and STDR in order to modify the current standards of care for T2D patients.

\section{REFERENCES}

1. Ramasamy C. Cardiologist-Dentist interaction in the dental management of immunocompromised cardiac patient. J Coll Physicians Surg Pak 2017; 27:261.

2. Liu Y, Song Y, Tao L, Qui W, Lv H, Jiang X, et al. Prevalence of diabetic retinopathy among 13,473 patients with diabetes mellitus in China: a cross-sectional epidemiological survey in six provinces. BMJ Open 2017; 7:e013199.

3. Mok Y, Tan CW, Wong HS, How CH, Tan KLA, Hsu PP. Obstructive sleep apnoea and type 2 diabetes mellitus: Are they connected? Singapore Med J 2017; 58:179-83.

4. Altaf QA, Dodson P, Ali A, Raymond NT, Wharton H, Fellows $\mathrm{H}$, et al. Obstructive sleep apnea and retinopathy in patients with type 2 diabetes. a longitudinal study. Am J Respir Crit Care Med 2017; 196:892-900.

5. Dawn Newspaper. Prof. Dr. Leszek Czupryniak. Pakistan has one of the highest rates of diabetes. Available at https:// www.dawn.com/news/1385369. Last accessed on January 26 , 2018.

Ramasamy Chidambaram

Department of Prosthodontics, AIMST University, Jalan Bedong-Semeling, 08100 Bedong, Kedah Darul Aman, Malaysia

Correspondence: Dr. Ramasamy Chidambaram, Department of Prosthodontics and Advance Conservative Dentistry, Faculty of Dentistry, AIMST University, Jalan BedongSemeling, 08100 Bedong, Kedah Darul Aman, Malaysia E-mail:dr.ramasamyc@gmail.com

Received: May 17, 2018; Accepted: August 02, 2018 …ㄷ…. 